\title{
Sofosbuvir and Ribavirin With or Without Pegylated Interferon for Hepatitis C Genotype 3: A Real World Experience
}

\author{
Zaigham Abbas, ${ }^{1,2,}$ Muhammad Saad, ${ }^{1}$ Ramlah Nadeem, ${ }^{2}$ Fahad Jawed, ${ }^{2}$ and Minaam Abbas ${ }^{2}$ \\ ${ }^{1}$ Department of Gastroenterology, Dr. Ziauddin University, Karachi 75500, Pakistan \\ ${ }^{2}$ Department of Medicine, Orthopedic and Medical Institute, Karachi 74400, Pakistan \\ "Corresponding author: Zaigham Abbas, FCPS, FRCP, Department of Gastroenterology, Dr. Ziauddin University Hospital, Karachi 75600, Pakistan. Tel: +92-2135862939, Fax: \\ +92-2135862940, E-mail: drzabbas@gmail.com
}

Received 2017 January 28; Revised 2017 February 26; Accepted 2017 March 14.

\begin{abstract}
Background: Documentation of the effectiveness of sofosbuvir and ribavirin with or without pegylated interferon alfa for hepatitis C virus Genotype 3 (HCV GT3) is limited in a real world setting.

Objectives: The present study aimed at examining the outcome of the above therapy in a real world setting.

Methods: Dual therapy of sofosbuvir and ribavirin was given for 24 weeks and triple therapy with additional pegylated interferon for 12 weeks. Patients received dual therapy when there was unwillingness to take interferon or interferon ineligibility.

Results: In this analysis, 241 patients were included, of whom 175 were treated with dual and 66 with triple therapy. The mean age of the patients was 46.6 years, ranging from 20 to 72 , and 136 (56.4\%) were male. Clinical cirrhosis was present in 151(62.7\%) patients, and 98 (40.7\%) had treatment experience. HCV RNA became negative in 225 (93.3\%) patients at week 4 of the treatment. End of treatment virologic response was observed in 221 (91.7\%) patients, and 199 (82.6\%) had sustained virologic response 12 weeks post treatment (SVR12). Undetectable HCV RNA at week 4 was an independent parameter predicting SVR12 $(\mathrm{P}=0.001)$. SVR12 was achieved in 143 (81.7\%) patients treated with dual therapy and $56(84.8 \%)$ with triple therapy $(\mathrm{P}=0.567)$. Prior HCV treatment status and presence or absence of cirrhosis did not significantly affect the outcome between the 2 treatment groups.

Conclusions: Treatment of HCV GT3 infection with the sofosbuvir and ribavirin with or without PEG-IFN $\alpha$ achieved durable responses in a significant number of cases in a real world setting.
\end{abstract}

Keywords: Chronic Hepatitis C, Sofosbuvir, Antiviral Agents, Ribavirin, Pegylated Interferon

\section{Introduction}

Pakistan has the most astounding number of persons infected with active hepatitis $\mathrm{C}$ virus (HCV) infection among all nations other than China (1). Yet, dissimilar to China, the aggregate number of infections is not declining. Most prevalent HCV genotype is 3 (GT3), which is present in $79 \%$ of cases $(2,3)$.

Direct-acting antiviral agents (DAA) are now the standard care in patients with chronic hepatitis $C$ infection (4). The current treatment recommendations for HCV GT3 are based on several phase $2 / 3$ clinical trials. Sofosbuvir, an NS5B polymerase inhibitor, has a pan-genotypic anti-HCV activity and emerged as an important component of currently available anti-HCV regimens. It has been shown that patients with Genotype 3 have a higher risk for all worse outcomes compared to Genotype 2 or 1 (5). In registration trials, the effect of sofosbuvir containing regimens has been modest among patients with GT3 infection, especially in those with cirrhosis. As indicated by the VALANCE phase III study, the response to sofosbuvir and ribavirin in Genotype 3 (GT3) patients after 24 weeks of treatment was 94\% (86/92) in non-cirrhotic treatment-naive persons, 92\%
(12/13) in cirrhotics treatment-naive, 87\% (87/100) in noncirrhotic treatment-experienced patients, and 60\% (27/45) in cirrhotic treatment-experienced patients (6), The results of the Boson study showed that the treatment response in GT3 may be improved if pegylated interferon alfa (PEGIFN $\alpha$ ) is combined with ribavirin, and treatment duration may be shortened to 12 weeks like other genotypes (7).

The real world data of treating GT3 patients with sofosbuvir-based regimens is limited. Sofosbuvir became available in Pakistan in 2014. Although Pakistan is a highly endemic area of HCV GT3 infection, no local study is available as the country was not chosen for clinical trials.

\section{Objectives}

The present study aimed at assessing the safety and efficacy of sofosbuvir containing regimes in a heterogeneous population of GT3 patients seen in daily practices. These patients were treated with sofosbuvir with ribavirin with or without pegylated interferon. 


\section{Methods}

This retrospective observational study shares the efficacy of sofosbuvir and ribavirin with or without PEG-IFN $\alpha$ in a real world setting. Duration of treatment was 24 weeks for dual therapy and 12 weeks for triple therapy with a posttreatment follow-up of 12 weeks. Treatment options were discussed with the patients. Triple therapy was offered to the interferon eligible patients. Patients who were not willing to receive pegylated interferon injections or interferon ineligible were given dual therapy.

The participants of this study were a diverse population of treatment naive and treatment experienced HCV GT3 patients, both nonresponders and relapsers to the PEGIFN $\alpha$ and ribavirin therapy. Diagnosis of clinical cirrhosis was based on clinical findings, laboratory parameters upper abdominal sonography, endoscopy and transient elastography in some cases. Inclusion criteria for starting treatment were age older than 18 years, reactive anti-HCV antibody, and measureable serum HCV RNA at enrolment by real time PCR, patients with compensated liver disease at the time of initiating treatment, hemoglobin $>11.0 \mathrm{~g} / \mathrm{dL}$ for males and $>10.0 \mathrm{~g} / \mathrm{dL}$ for females at screening, total leucocyte count $>3.0 \times 10^{9} / \mathrm{L}$, and neutrophils $>1.5 \times 10^{9} / \mathrm{L}$, platelets $>50 \times 10^{9} / \mathrm{L}$ for dual therapy and $80 \times 10^{9} \mathrm{~L}$ for triple therapy, serum creatinine level $<1.5 \mathrm{mg} / \mathrm{dL}$, will of receiving and adhering to the treatment, and no contraindications to the therapy.

Exclusion criteria were patients coinfected with hepatitis B or human immunodeficiency virus, decompensated liver disease at the time of initiation of treatment, history or proof of a medical condition associated with chronic liver disease (eg, toxin exposures, thalassemia, hemochromatosis, Wilson's disease, Alpha1 antitrypsin deficiency), autoimmune hepatitis, alcoholic liver disease, pregnant or breast feeding women, alcohol or drug abuse, and severe cardiopulmonary disease.

Sofosbuvir (Sovaldi, Gilead Sciences, Inc. Foster City, CA 94404) was given in a dose of $400 \mathrm{mg}$ per day, and ribavirin dose was $500 \mathrm{mg}$ twice a day for patients less than $75 \mathrm{~kg}$ of body weight and $600 \mathrm{mg}$ twice a day for patients over $75 \mathrm{~kg}$. The dose of PEG-IFN $\alpha$-2a (Ropegra, F. Hoffmann-La Roche Ltd, Basel) was $180 \mu$ g weekly ad for PEG-IFN $\alpha$-2b (PegIntron Merck and Co., Inc., Whitehouse Station, NJ 08889, USA) 1.5 $\mu \mathrm{g} / \mathrm{kg}$ of body weight.

Primary endpoint was negative HCV RNA12 weeks after the completion of treatment(SVR12). Secondary end points were rapid viral response (RVR), HCV- PCR below the detectable limits at 4 weeks after initiation of treatment and end of treatment response (ETR), and HCV- PCR below the detectable limits at the end of the treatment. Treatment failure was defined as nonresponse; ie, detectable HCVRNA at the end of treatment. Relapse was defined as reappearance of HCV RNA at 12 weeks post treatment in patients who achieved ETR. Stopping treatment because of any reason, lost to follow- up, and dropouts were considered as treatment failure (Figure 1).

The patients were instructed with respect to administration of oral treatment and subcutaneous pegylated interferon, predictable adverse events, timetable for laboratory monitoring, and clinic follow-up. Patients were assessed as outpatients for wellbeing, side effects, and adequacy at regular intervals amid treatment every 4 weeks during treatment, end of treatment, and at 12 weeks posttreatment. At each visit, biochemistry and complete blood counts were assessed. RNA was extracted from the specimens and examined using COBAS TaqMan Analyzer v2. 0 (Roche Molecular Systems, Inc., Branchburg, NJ, 08876 USA) with a lower cutoff of recognition $15 \mathrm{IU} / \mathrm{mL}$. HCV RNA levels were measured at baseline, 4 weeks, end of treatment, and end of follow-up.

Adverse events and laboratory parameters were noted. A drop of $\mathrm{Hb} \%$ more than $2 \mathrm{~g} / \mathrm{dL}$ within 8 weeks of initiation of antiviral therapy was managed by erythropoietin, followed by dose reduction of ribavirin if needed. After 8 weeks of antiviral therapy, ribavirin dose reduction was tried first, followed by erythropoietin if needed. A drop of neutrophil count to less than $1000 / \mathrm{mm} 3$ was dealt by subcutaneous filgrastim. The platelet count of 25,000 $50,000 / \mathrm{mm}^{3}$ was managed by interferon dose reduction.

\subsection{Statistical Analysis}

Evidences were stated as the number of participants with percentages for nominal variables and were compared by chi-square or Fisher's exact test. Continuous variables were displayed as mean with standard deviation, and compared using Mann-Whitney U test. Binary logistic regression analysis was used to define independent factors associated with SVR12. Statistical analyses were conducted using SPSS 23.0 software (IBM SPSS Statistics, New York, NY, United States). All tests were 2-tailed and a P value $<0.05$ was set for statistical significance.

\section{Results}

Among HCV GT3 treated patients during 2015 and 2016, 241 patients reached the end of analysis at the time of writing this manuscript, 175 were treated with sofosbuvir and ribavirin, and 66 patients with sofosbuvir, ribavirin, and PEG-IFNa. The mean age of the patients was 46.6 years, ranging from 20 to 72; of the patients, 136 (56.4\%) were male, clinical cirrhosis was present in 151 (62.7\%), and 98 patients were treatment experienced (40.7\%). Baseline investigations were as follow: haemoglobin $12.9 \pm 1.9 \mathrm{~g} / \mathrm{dL}$, 


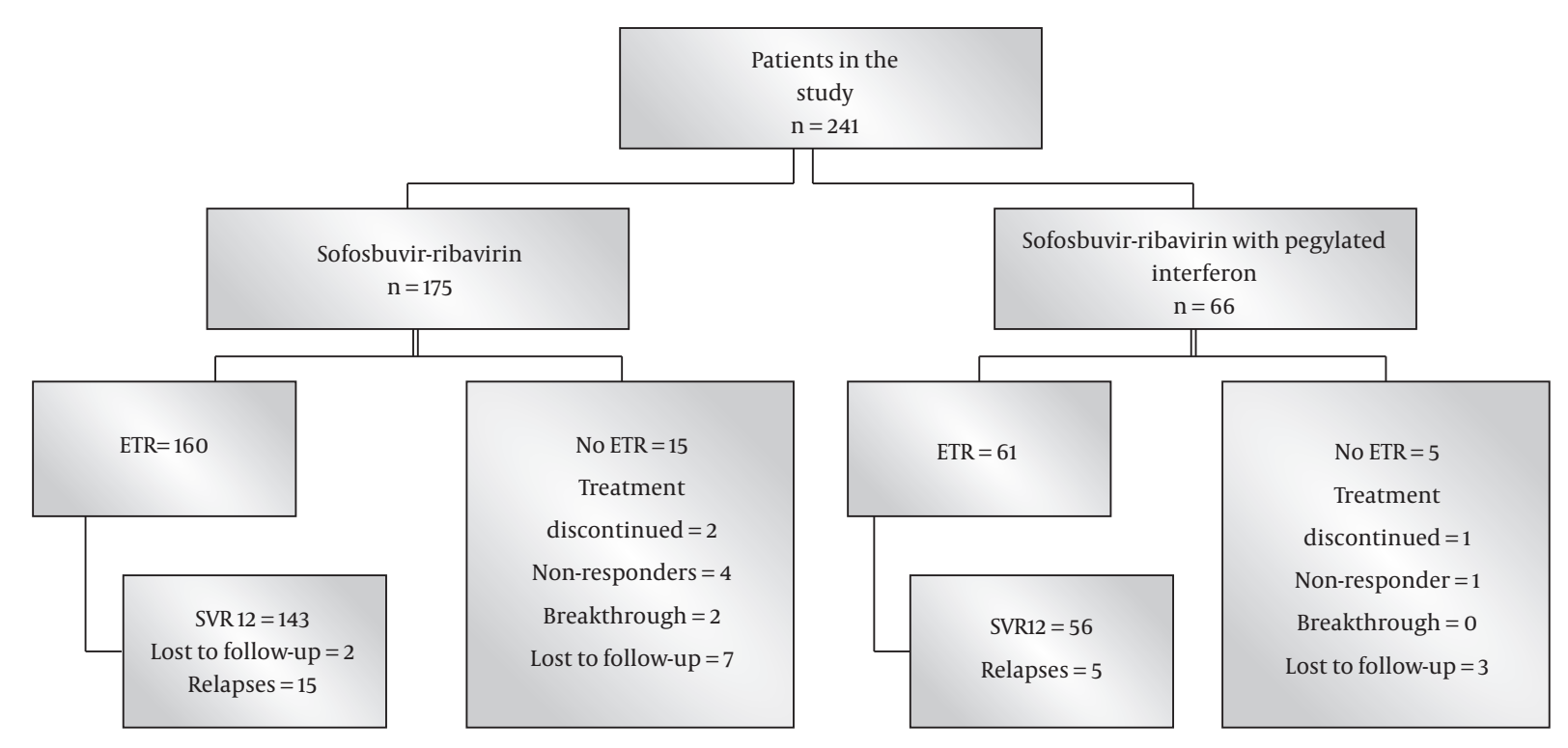

Figure 1. Flow Diagram of the Study Patients

leucocytes $6.1 \pm 2.1 \times 10^{9} / \mathrm{L}$, platelets $166 \pm 91 \times 10^{9} / \mathrm{L}$, total bilirubin $1.01 \pm 0.7 \mathrm{mg} / \mathrm{dL}$, alanine aminotransferase (ALT) $77 \pm 51 \mathrm{IU} / \mathrm{L}$, gamma glutamyl transferase (GGT) $94 \pm 91$ IU/L, alkaline phosphatase $13 \pm 70 \mathrm{IU} / \mathrm{L}$, and HCV RNA 5.17 $\pm 0.99 \log 10 / \mathrm{mL}$. HCV RNA became negative in 225 (93.3\%) patients at week 4 of treatment. ETR was observed in 221 (91.7\%) and SVR12 in 199 (82.6\%) patients. ALT was normal in 193 (80.1\%) patients at the end of the follow-up.

Demographic and baseline clinical characteristics of the patients in the 2 groups and response to the treatment are demonstrated in Table 1 . As expected, triple therapy, which included pegylated interferon, was avoided in patients with advanced disease or a history of previous decompensation. There was no noteworthy difference in the response towards the end of treatment and end of followup. Between the 2 groups, SVR12 rates did not differ significantly in patients with or without cirrhosis or a history of previous treatment. Combining data of the 2 groups, treatment naive status, absence of clinical cirrhosis, and undetectable HCV RNA at week 4 were associated with SVR12. Binary logistic regression analysis maintained undetectable HCV RNA at week 4 as an independent parameter predicting SVR12 $(\mathrm{P}=0.001)$.

The common adverse effects experienced by the patients who received the sofosbuvir-ribavirin therapy were fatigue (21.7\%), body aches (13.7\%), dyspepsia (12.5\%), fever (6.8\%), insomnia (5.7\%), and headache (5.1\%). Common adverse effects of sofosbuvir-ribavirin plus pegylated interferon were body aches (24\%), dyspepsia (18\%), fatigue (15\%), and fever (15\%). These side effects did not require a dose re- duction. Serious adverse events were reported more in patients receiving dual therapy because this group included patients with a history of previous decompensation. Anemia requiring erythropoietin and transient dose reduction of ribavirin was observed in 10 (5.7\%) patients with dual therapy and 1 (1.5\%) patient with triple therapy. One patient on dual therapy with severe anemia received blood transfusion, and 7 patients (10.6\%) in the triple therapy group required filgrastim to treat transient leucopenia. Severe thrombocytopenia occurred in $3(1.7 \%)$ patients with dual therapy and 4(6\%) with triple therapy, 9 (5.1\%) developed ascites, and $2(1.1 \%)$ hepatic encephalopathy in the dual therapy group. Mild ascites and encephalopathy were managed with diuretics and lactulose, respectively. Therapy was discontinued in 2 patients who received dual therapy, 1 patient developed advanced encephalopathy, and the second one severe ascites. Treatment was stopped in 1 patient who received triple therapy due to severe thrombocytopenia.

\section{Discussion}

Studies that evaluated sofosbuvir with ribavirin given for 12 weeks among different GT3 patient groups had SVR12 rates of $19 \%$ to $56 \%(8,9) 8.9$. Response rates were lower in patients with cirrhosis compared to noncirrhotic patients. In the VALENCE trial by Zeuzem et al. $85 \%$ of the patients had SVR12 when sofosbuvir and ribavirin were given for 24 weeks (6). Response rates were 91\% and 68\% among 
Table 1. Baseline Characteristics of the Study Patients and Response to Treatment

\begin{tabular}{|c|c|c|c|}
\hline & Sofosbuvir-Ribavirin $(n=175)$ & $\begin{array}{l}\text { Sofosbuvir-Ribavirin Plus Pegylated Interferon }(\mathbf{n}= \\
66)\end{array}$ & P Value \\
\hline \multicolumn{4}{|l|}{ Baseline characteristics of the patients } \\
\hline Mean age, $y$ & $48.1 \pm 11.3$ & $42.5 \pm 10.4$ & $0.000^{\mathrm{b}}$ \\
\hline Mean weight, kg & $69.5 \pm 15.5$ & $68.0 \pm 14.9$ & 0.728 \\
\hline Male gender & $99(56.6)$ & $37(56.1)$ & 0.943 \\
\hline Treatment naive patients & $101(57.7)$ & $42(63.6)$ & 0.404 \\
\hline Presence of clinical cirrhosis & $123(70.2)$ & $28(42.4$ & $0.000^{\mathrm{b}}$ \\
\hline Past history of decompensation & $38(21.7)$ & $2(3.0)$ & $0.000^{\mathrm{b}}$ \\
\hline Hemoglobin, g/dL & $12.8 \pm 1.9$ & $13.3 \pm 1.9$ & 0.059 \\
\hline Total leukocyte count, $\times 10^{9} / \mathrm{L}$ & $6.0 \pm 2.0$ & $6.5 \pm 2.1$ & 0.115 \\
\hline Platelets, $\times 10^{9} / \mathrm{L}$ & $153 \pm 91$ & $200 \pm 79$ & $0.000^{\mathrm{b}}$ \\
\hline Total bilirubin, mg/dL & $1.1 \pm 0.7$ & $0.8 \pm 0.6$ & $0.007^{\mathrm{b}}$ \\
\hline ALT, IU/L & $72 \pm 46$ & $88 \pm 60$ & 0.140 \\
\hline $\begin{array}{l}\text { ALT within the upper limit of normal before } \\
\text { starting treatment }\end{array}$ & $43(24.6)$ & $12(18.2)$ & 0.292 \\
\hline GGT, IU/L & $93 \pm 93$ & $96 \pm 88$ & 0.713 \\
\hline Alkaline phosphatase, IU/L & $135 \pm 77$ & $119 \pm 51$ & 0.448 \\
\hline HCV RNA, $\log 10 \mathrm{IU} / \mathrm{mL}$ & $5.6 \pm 1.1$ & $5.6 \pm 0.8$ & 0.543 \\
\hline \multicolumn{4}{|l|}{ Virologic and biochemical responses } \\
\hline Undetectable HCV RNA at week 4 & $154(88.0)$ & $63(95.4)$ & 0.096 \\
\hline End of treatment virologic response & $160(91.4)$ & $61(92.4)$ & 0.803 \\
\hline Sustained virologic response (SVR) & $143(81.7)$ & $56(84.8)$ & 0.567 \\
\hline Treatment naive patients & $90 / 101(89.1)$ & $37 / 42(88.1)$ & 0.861 \\
\hline Treatment experienced patients & $53 / 74(71.6)$ & $19 / 24(79.2)$ & 0.467 \\
\hline Cirrhotics & $95 / 123(77.2)$ & $23 / 28(82.1)$ & 0.571 \\
\hline Non-cirrhotics & $48 / 52(92.3)$ & $33 / 38(86.8)$ & 0.485 \\
\hline Non-cirrhotics and treatment naive & $33 / 36(91.7)$ & $25 / 28(89.3)$ & 1.000 \\
\hline Non-cirrhotics and treatment experienced & $15 / 16(93.7)$ & $8 / 10(80.0)$ & 0.538 \\
\hline Cirrhotic treatment naive & $57 / 65(87.7)$ & $12 / 14(85.7)$ & 1.000 \\
\hline Cirrhotic treatment experienced & $38 / 58(65.5)$ & $11 / 14(78.6)$ & 0.525 \\
\hline ALT within ULN at the end of treatment & $147(84.0)$ & $50(75.7)$ & 0.140 \\
\hline ALT within ULN at the end of follow-up & $139(79.4)$ & $54(81.8)$ & 0.679 \\
\hline
\end{tabular}

Abbreviations: ALT, alanine aminotransferase; GGT, gamma glutamyl transferase; HCV, Hepatitis C virus; RNA, Ribonucleic Acid.

${ }^{\mathrm{a}}$ Values are expressed as mean $\pm \mathrm{SD}$ or No. $(\%)$.

${ }^{\mathrm{b}}$ Significant Values.

those without and with cirrhosis. As evident by these trials, the basic problem was to deal with the treatment of patients with cirrhosis, especially those who had already been treated before. In our study, in those patients who received sofosbuvir and ribavirin dual therapy $(n=175)$, the response rate was $77.2 \%$ among patients with cirrhosis compared to $92.3 \%$ who did not have clinical cirrhosis $(\mathrm{P}=$
0.019). These better results from the dual therapy in our patients with cirrhosis are promising as this group had a significant number of patients who had a previous history of decompensation. Whether the better response was due to ethnicity or the response differed in higher endemic areas for GT3 need to be further evaluated.

The Boson study showed that the triple therapy of so- 
fosbuvir, ribavirin, and PEG-IFN $\alpha$ for 12 weeks was better than dual therapy of sofosbuvir and ribavirin given for 24 weeks in all categories of GT3 infected patients (7). SVR12 was $84 \%$ among patients receiving 24 weeks of sofosbuvir and ribavirin, and 93\% in those receiving sofosbuvir, ribavirin, and PEG IFN $\alpha$. In treatment experienced cirrhotic patients, SVR12 decreased to $77 \%$ in patients who received dual therapy compared to $86 \%$ who received triple therapy. In our study, SVR12 were $81.7 \%$ among all patients who received dual therapy and $84.8 \%$ patients who received triple therapy. Lower response rates in our cohort may be due to the presence of a large proportion of patients with cirrhosis. Response rates decreased to $65.5 \%$ in our treatment experienced cirrhotic patients who received dual therapy compared to $78.6 \%$ who received triple therapy. However, it could not reach a statistically significant difference perhaps due to a smaller number of patients in the triple therapy group. Thus, our data could not replicate similar superior results for all categories of triple therapy recipients. A recent real world study has a similar conclusion; however, their sample size for GT3 patients was much smaller than our study (10).

Although triple therapy is of shorter duration, it cannot be offered to patients who had previously decompensated or where hematology parameters are unfavourable for interferon therapy. Acceptance of injection based therapy is low due to hatred and fear for injections and its side effects. The option of interferon- based therapy can only be used for motivated interferon eligible patients. Moreover, the acceptance of injection based regimen is expected to be low in pegylated interferon treatment experienced patients due to reminiscences of adverse events. This has led to the asymmetry between the 2 treatment groups in our study as well. Ribavirin is again not a good drug due to its major side effect of causing anemia. More data are needed to formulate guidance for GT3 patients in our setting because SVR rates remain $<90 \%$ from available regimens, highlighting the need for better options. Recent strategies are to add on additional DAAs to sofosbuvir including velpatasvir, daclatasvir, or grazoprevir plus elbasvir (1113). Current AASLD / IDSA guidance has already mentioned that both of our study regimens may not be recommended when newly approved DAAs are available (4). Because sofosbuvir is the only DAA registered in our country, it will continue to be used in our and many other countries. The cost will be another issue even when other DAAs become available.

The sofosbuvir and ribavirin combination with or without PEG-IFN $\alpha$ showed a fair safety profile in our both cirrhotic and noncirrhotic patients. The side effects were manageable in most of the cases. Although decompensation occurs in some patients, our cohort had a good num- ber of patients with previous history of decompensation. Ribavirin related anemia was managed either by giving erythropoietin or reducing the dose. Recurrence of ascites was managed by addition of diuretics. Thus, closer monitoring may be needed for patients with advanced cirrhosis and portal hypertension.

The development of DAA has dramatically improved tolerability of treatment and cure rates. With the availability of sofosbuvir, we were able to manage patients who could not be treated previously due to medical or psychiatric contraindications or an inability to tolerate pegylated interferon. Elimination of hepatitis $\mathrm{C}$ is now feasible in the next 2 decades through an aggressive national strategy that combines prevention, screening, and treatment. With this aggressive approach, 90\% reduction in the total number of viremic individuals is expected by 2030 (14). This is only possible if safe and potent newer DAAs would be available at affordable prices.

In conclusion, overall SVR12 in the real world HCV GT3 patients was $82.6 \%$ across 2 regimens and various patient groups. SVR12 was achieved in 143 (81.7\%) patients with dual therapy and 56 (84.8\%) with triple therapy. Both regimens were effective and well- tolerated by patients. Between the 2 groups, SVR12 rates did not differ significantly in patients with or without cirrhosis or a history of previous treatment. However, as it was an analysis of nonrandomized data, our comparison of SVR rates between the 2 regimens had its limitations. The therapy achieved a lower SVR12 compared to ideal treatment outcomes of greater than $90 \%$. Treatment-experienced cirrhotic GT3 patients are among the most difficult to treat. Use of PEGIFN $\alpha$ may be considered in the compensated disease until effective and affordable DAA combinations become available. Our findings highlight the need for establishing alternative treatment strategies for HCV GT3 patients.

\section{Footnote}

Conflict of Interest: The authors declare no potential conflict of interest.

\section{References}

1. Gower E, Estes C, Blach S, Razavi-Shearer K, Razavi H. Global epidemiology and genotype distribution of the hepatitis C virus infection.J Hepatol. 2014;61(1Suppl):S45-57. doi:10.1016/j.jhep.2014.07.027. [PubMed: 25086286].

2. Attaullah S, Khan S, Ali I. Hepatitis C virus genotypes in Pakistan: a systemic review. Virol J. 2011;8:433. doi: 10.1186/1743-422X-8-433. [PubMed: 21902822].

3. Aghemo A, Dore GJ, Hatzakis A, Wedemeyer H, Razavi H. Estimating HCV disease burden - volume 3 (editorial). JViral Hepat. 2015;22 Suppl 4:1-3. doi: 10.1111/jvh.12473. [PubMed: 26513444]. 
4. Aasld Idsa Hcv Guidance Panel . Hepatitis C guidance: AASLD-IDSA recommendations for testing, managing, and treating adults infected with hepatitis C virus. Hepatology. 2015;62(3):932-54. doi: 10.1002/hep.27950. [PubMed: 26111063].

5. Kattakuzhy S, Levy R, Rosenthal E, Tang L, Wilson E, Kottilil S. Hepatitis C genotype 3 disease. Hepatol Int. 2016;10(6):861-70. doi: 10.1007/s12072-016-9748-z. [PubMed: 27328848].

6. Zeuzem S, Dusheiko GM, Salupere R, Mangia A, Flisiak R, Hyland RH, et al. Sofosbuvir and ribavirin in HCV genotypes 2 and 3. $N$ Engl $J$ Med. 2014;370(21):1993-2001. doi: 10.1056/NEJMoa1316145. [PubMed: 24795201].

7. Foster GR, Pianko S, Brown A, Forton D, Nahass RG, George J, et al. Efficacy of sofosbuvir plus ribavirin with or without peginterferonalfa in patients with hepatitis $C$ virus genotype 3 infection and treatment-experienced patients with cirrhosis and hepatitis $C$ virus genotype 2 infection. Gastroenterology. 2015;149(6):1462-70. doi: 10.1053/j.gastro.2015.07.043. [PubMed: 26248087].

8. Lawitz E, Mangia A, Wyles D, Rodriguez-Torres M, Hassanein T, Gordon SC, et al. Sofosbuvir for previously untreated chronic hepatitis $\mathrm{C}$ infection. N Engl J Med. 2013;368(20):1878-87. doi: 10.1056/NEJMoa1214853. [PubMed: 23607594].

9. Jacobson IM, Gordon SC, Kowdley KV, Yoshida EM, Rodriguez-Torres M, Sulkowski MS, et al. Sofosbuvir for hepatitis C genotype 2 or 3 in patients without treatment options. N Engl J Med. 2013;368(20):186777. doi: 10.1056/NEJMoa1214854. [PubMed: 23607593].

10. Satsangi S, Mehta M, Duseja A, Taneja S, Dhiman RK, Chawla Y. Dual treatment with sofosbuvir plus ribavirin is as effective as triple therapy with pegylated interferon plus sofosbuvir plus ribavirin in predominant genotype 3 patients with chronic hepatitis C. J Gastroenterol Hepatol. 2017;32(4):859-63. doi: 10.1111/jgh.13595. [PubMed: 27624314].

11. Younossi ZM, Stepanova M, Sulkowski M, Foster GR, Reau N, Mangia A, et al. Ribavirin-Free Regimen With Sofosbuvir and Velpatasvir Is Associated With High Efficacy and Improvement of Patient-Reported Outcomes in Patients With Genotypes 2 and 3 Chronic Hepatitis C: Results From Astral-2 and -3 Clinical Trials. Clin Infect Dis. 2016;63(8):1042-8. doi: 10.1093/cid/ciw496. [PubMed: 27444413].

12. Nelson DR, Cooper JN, Lalezari JP, Lawitz E, Pockros PJ, Gitlin N, et al. All-oral 12-week treatment with daclatasvir plus sofosbuvir in patients with hepatitis C virus genotype 3 infection: ALLY-3 phase III study. Hepatology. 2015;61(4):1127-35. doi: 10.1002/hep.27726. [PubMed: 25614962].

13. Poordad F, Lawitz E, Gutierrez JA, Evans B, Howe A, Feng HP, et al. 0006 : C-swift: grazoprevir/elbasvir + sofosbuvir in cirrhotic and noncirrhotic, treatment-naive patients with hepatitis $C$ virus genotype 1 infection, for durations of 4,6 or 8 weeks and genotype 3 infection for durations of 8 or 12 weeks. J Hepatol. 2015;62:S192-3. doi: 10.1016/s01688278(15)30013-1.

14. Alfaleh FZ, Nugrahini N, Maticic M, Tolmane I, Alzaabi M, Hajarizadeh $B$, et al. Strategies to manage hepatitis $C$ virus infection disease burden - volume 3. J Viral Hepat. 2015;22 Suppl 4:42-65. doi 10.1111/jvh.12474. [PubMed: 26513447]. 